\section{Analyse de thèse}

Orthop Traumatol (1992) $2: 50$
ORTHOPÉDIE
TRAUMATOLOGIE

(C) Springer-Verlag 1992

\section{N. Armbruster \\ La méthode de Ferguson dans la maladie luxante de la hanche chez le nourrisson et le nouveau-né. A propos de 82 cas \\ Thèse Strasbourg, 1992, $n^{\circ} 8$}

La maladie luxante de hanche est dépistée de plus en plus précocement, faisant disparaître le diagnostic à l'âge de la marche. Or la première année de vie est la période privilégiée pour le pronostic et le traitement. L'auteur a donc voulu comparer :

- Un traitement, classiquement orthopédique (traction, culotte d'Ortolani, harnais de Pavlik), mais long, lent, succeptible d'être complété par une chirurgie tardive sur une dysplasie résiduelle.

-Un traitement chirurgical précoce (opération de Ferguson) dont la section du psoas, l'ouverture du sablier capsulaire, et le nettoyage de la cavité articulaire permet un centrage précis de la tête favorisant le remodelage ultérieur.

Ainsi 75 enfants répartis en deux groupes selon la présence ou l'absence de traitement préalable furent étudiés.

Si la réduction concentrique est obtenue dans tous les cas, seulement $55 \%$ des enfants ont des résultats coxométriques normaux à la fin du traitement. La complication principale est l'ostéochondrite (6\%), semblant essentiellement liée au traitement orthopédique mal conduit (abduction trop forte).

L'échec de la méthode (reluxation) est liée à l'inexpérience, un âge supérieur à 9 mois, ou la gravité de la dysplasie cotylö̈dienne (angle $\mathrm{C}>40^{\circ}$ ).

L'insuffisance de traitement apparait secondairement à une coxa-valga (16\%), une antétorsion trop forte ou la persistance de la dysplasie cotyloidienne $(20 \%)$.

Comparativement au Harnais de Pavlik, la réduction n'est pas obtenue à $100 \%$, nécessitant une réduction longue et progressive (flexion $>90^{\circ}$, abduction correcte) avec la compréhension des parents. En cas d'échec le diagnostic est tardif ( 3 à 4 mois) retardant d'autant la réduction et la correction de la dysplasie. 